\title{
Beyond the Langevin Horn: Transducer Arrays for the Acoustic Levitation of Liquid Drops
}

\author{
Robert H. Morris ${ }^{1 *}$, Elizabeth R. Dye ${ }^{1}$, Peter Docker ${ }^{2}$, Michael I. Newton ${ }^{1}$ \\ ${ }^{1}$ School of Science and Technology, Nottingham Trent University, Nottingham, NG11 8NS, \\ UK \\ ${ }^{2}$ Diamond Light Source, Harwell Science and Innovation Campus, Oxfordshire, OX11 ODE, \\ UK \\ *rob.morris@ntu.ac.uk
}

\begin{abstract}
The acoustic levitation of liquid drops has been a key phenomenon for more than 40 years, driven partly by the ability to mimic a microgravity environment. It has seen more than 700 research articles published in this time and has seen a recent resurgence in the past 5 years thanks to low cost developments. As well as investigating the basic physics of levitated drops, acoustic levitation has been touted for container free delivery of samples to a variety of measurements systems, most notably in various spectroscopy techniques including Raman, Fourier Transform InfraRed (FTIR) in addition to numerous X-Ray techniques. For 30 years the workhorse of the acoustic levitation apparatus was a stack comprising a piezoelectric transducer coupled to a horn shaped radiative element often referred to as the Langevin horn. Decades of effort have been dedicated to such devices, paired with a matching and opposing device or a reflector, but they have a significant dependence on temperature and require precision alignment. The last decade has seen a significant shift away from these in favour of arrays of digitally driven, inexpensive transducers, giving a new dynamic to the topic which we review herein.
\end{abstract}

\section{Introduction}

Levitation has been used for many years to manipulate materials within air and liquid environments without physical interaction with the container surface. It aims to create a micro gravity environment to study the materials which are levitated. There are many forms of levitation including acoustic, magnetic, electrostatic, optical and aerodynamic.

Suspension against gravity using magnetic levitation requires either the sample to be ferromagnetic, or for the magnetic fields to be amongst the largest produced on Earth in order to levitate diamagnetic materials which has for example, been used to levitate a frog [1]. Electrostatic levitation uses an electric field to levitate the sample, requiring a sample which can, and must, be charged in order to achieve suspension against gravity [2]. Optical levitation has only been achieved for very small and lightweight particles, as it is achieved by firing a focused laser beam at the sample and utilising the transfer of momentum from the photons to the sample surface. For this method, the refractive index of the particle must be higher than that of the medium it is suspended within, limiting this technique to a very small number of cases [3]. Finally, aerodynamic levitation is achieved using a high-pressure gas jet to suspend the material under investigation, but this causes significant agitation and may alter the sample in question [4]. In contrast, acoustic levitation is for a wide range of cases is a superior presentation method, as the variety of materials which may be levitated is far greater since there is no requirement for magnetic or chargeable samples. These systems 
use an emitter and reflector, or multiple emitters, that produce frequencies above that which can be heard by an average human, to create a series of positions in which the conditions are suitable to entrap particles or sample droplets.

Previous review articles have extensively covered the core topics of acoustic levitation: Work by Brandt [6] has covered the principles of the different types of levitation, whereas Andrade et al. [7] completed a comprehensive review of acoustic levitation. Santesson et al. [8] have also published a review to inform the use of acoustic levitators within chemistry, describing the various processes which can be aided by its use. These reviews have however mainly covered the use of traditional acoustic levitation systems primarily utilising the Langevin horn. Although a key source of ultrasonic radiation, they often require more than 1000 volts to power them at powers over $130 \mathrm{~W}$ and often cause heating to the sample which is suspended $[9,53]$. The modern trend towards arrays of cheaper low power off-theshelf transducers achieve similar suspension forces to some Langevin horns but with negligible heating of the sample and typically a power supply of $12-15 \mathrm{~V}$ at powers less than 10W [39].

Acoustic levitation has been well demonstrated as a technique for the containerless suspension of samples for remote analysis. It has been used in synchrotron [10 - 13], x-ray [14 - 16] and Raman spectroscopy [17 - 19] experiments. A range of additional spectroscopy experiments have also been performed, including FTIR [20 - 24], X-Ray Spectroscopy [25], fluorescence spectroscopy [26] and mass spectroscopy [27 - 29].

The current state of the art for spectroscopic analysis of levitated liquid droplets using a conventional piezoelectric horn levitator is reported by Brotton et al. $[21,23]$. In this work a piezoelectric transducer oscillates at $58 \mathrm{kHz}$ (using $v=f \lambda$ and the speed of sound in air at STP, yields a wavelength in air of about $5.9 \mathrm{~mm}$ ). In the earlier of these two papers, the largest diameter of particles that could be levitated was approximately $2.5 \mathrm{~mm}$, whereas the smallest was around $15 \mu \mathrm{m}$. In the later paper, the size claimed was up to $3 \mathrm{~mm}$ which is at the half wavelength diameter limit. Their measurement system combined Raman, near-IR, UV-vis and FTIR spectroscopies within the same measurement chamber that also allowed laser heating of the sample droplet. Owing to the small total heat capacity, the levitated particle can be heated to a high temperature and cooled over very short time scales thus allowing for precise control of the sample temperature. Typical of the state of the art for Xray diffraction of levitated droplets is reported by Tsujino et al. [13] at the X06SA beamline at the Swiss Light Source. Their levitation system operated at around $38 \mathrm{kHz}$ (corresponding to a wavelength in air of $9 \mathrm{~mm}$ ). Rapid spinning of the crystal orientation inside the droplet, which is typical of levitated drops, meant that additional instrumentation for sample oscillation and rotation, typically used with standard crystallography were not required. Typically using a $4 \mu \mathrm{l}$ droplet, consistent with the smallest size droplets reported by Brotton et al, a dataset of 3,600 diffraction images per run could be collected in a total duration of around $30 \mathrm{~s}$. These parameters define the range which are needed for phased arrays to compete with the best of the Langevin horn systems for presenting liquid droplets to measurement systems.

This article reviews the acoustic levitation methods which utilise transducer arrays to levitate and manipulate objects within air and their use as a sample suspension or delivery 
method for measurement systems. It follows the technological development journey from levitating expanded polystyrene particles to levitating droplets through applications.

\section{Fundamental Physics of Acoustic Levitation}

The reader is directed to comprehensive reviews on the physics of acoustic levitation for thorough treatment of the background physics. However, in the interests of completeness and to ensure that following discussions are fully accessible, the essential analysis of the acoustic force which suspends the samples against gravity is briefly discussed here.

Although the physical embodiment of an acoustic levitator may be highly complex, there are relatively few parameters needed to describe the so-called acoustic radiation force which describes the acoustic force exerted on a levitated sample. There are two primary approaches to this analysis which are discussed here. Gor'kov's expression is most often used for evaluation of small sample levitation in focal point systems such as Marzo's TinyLev, estimating the force upon a spherical particle in an arbitrary acoustic field within an ideal fluid. It considers the compressibility of the particle and that it may be set into motion due to the incident wave. In order to apply this expression, it is assumed that the radius of the spherical particle is much smaller than $\lambda$, the wavelength of the longitudinal wave which in many applications is on the order of $1-10 \mathrm{~mm}$. It should be noted that for phased arrays which are the primary focus of this review article, the frequency of the transducers (which determines the wavelength) is often based on availability of mass-produced transducers used for ultrasonic range finding or level detection which are typically $38-40 \mathrm{kHz}$.

To determine the acoustic radiation force, it is first necessary to calculate the time-averaged potential $U$, as follows in [EQ1]:

$$
U=2 \pi R^{3}\left[\left(\overline{p_{i n}^{2}} / 3 \rho c^{2}\right) f_{1}-\left(\rho \overline{v_{i n}^{2}} / 2\right) f_{2}\right]
$$

where $\mathrm{R}$ is the radius of the spherical particle, $\overline{p_{i n}^{2}}$ and $\overline{v_{i n}^{2}}$ are the mean-square fluctuations of the pressure and velocity respectively, at the point of the wave's interaction with the particle.

The factors $f_{1}$ and $f_{2}$ are described by [EQ2]:

$$
f_{1}=1-\rho c^{2} / p_{s} c_{s}^{2}, \quad f_{2}=2\left(\rho_{s}-\rho\right) /\left(2 \rho_{s}+\rho\right)
$$

where $\rho$ is the density of the fluid, $\rho_{s}$ is the density of the particle whilst $c$ and $c_{s}$ are the speeds of sound within the fluid and particle respectively.

The acoustic radiation force acting upon the particle may then be obtained from this result by finding the gradient of the potential [5].

This approach is however not applicable to the levitation of large samples, and an alternative approach is needed to evaluate broad array's such as used in haptic systems. This analysis requires determination of the acoustic radiation pressure $\mathrm{P}$ by assuming a plane wave as can be seen in equation EQ3. 


$$
P=\alpha E=\alpha \frac{I}{v}=\alpha \frac{p^{2}}{\rho v^{2}}
$$

where the ultrasound energy density is represented by $E, l$ is the sound intensity, the speed of sound in air is given by $v, p$ is the RMS ultrasound pressure of ultrasound and $\rho$ is the air density. Finally, $\alpha$ is a constant between 1 to 2 which scales the resulting pressure to account for the reflectivity of the levitated object with a value of 1 being complete absorption and 2 being complete reflection. Using this relationship, it can be seen that by manipulating the spatial distribution of the ultrasound pressure, the acoustic radiation pressure can be controlled to provide a desired distribution for a given sample.

\section{The Polystyrene Particle Years}

Levitation of liquid drops poses a significant challenge owing to the plethora of sample properties which dictate droplet shape. As a consequence, the levitation of expanded polystyrene particles has often heralded the introduction of a novel technique that has later been refined to accommodate liquids. Indeed, levitation was not the aim of much of the work that lead to these developments but a key development step in the production of a new generation of holographic display technologies. In Noncontact Tactile Display Based on Radiation Pressure of Airborne Ultrasound [30] Hoshi et al. demonstrated an array of ultrasonic transducers that allowed users to feel virtual objects in air, giving tactile feedback without any mechanical contact. Their prototype consisted of an array of $324,40 \mathrm{kHz}$ ultrasound transducers where the phase and intensity of each transducer were controlled individually based on the analysis of EQ3 to generate an acoustic force of $16 \mathrm{mN}$ over $20 \mathrm{~mm}$. The same group went on to develop this into a series of acoustic levitation devices, the first of which was reported in the 2014 publication Three-dimensional noncontact manipulation by opposite ultrasonic phased arrays [31] in which two arrays of ultrasonic transducers were arranged opposite each other to generate a localized standing wave at arbitrary positions utilising the so called phased-array focusing technique. This technique generates a focal point at a specific position by determining the path difference between the 0 -th and $n$-th transducers and using the speed of sound within air to find an appropriate time delay as given by equation [EQ3].

$$
T_{n}=\frac{d_{n}}{c}
$$

By delaying the start of the square wave signal to the $\mathrm{n}$-th transducer by this amount, the focal point is generated.

Three advantages were identified to such airborne ultrasound focusing device (AUFD) arrays:

- The particles can be manipulated in all directions according to the movement of the localized standing wave based on the phase-delay control,

- The work space is much larger than those in previous research studies because the ultrasound wave is focused and hence delivered farther and

- The particles are kept trapped even when the acoustic axis is horizontal because the AUFDs provide a sufficient amplitude of ultrasound. 
In Three-Dimensional Mid-Air Acoustic Manipulation by Ultrasonic Phased Arrays [32] the same group utilised four arrays of transducers at $40 \mathrm{kHz}$ or $25 \mathrm{kHz}$ to provide threedimensional control of expanded polystyrene particles up to $2 \mathrm{~mm}$ in diameter as can been seen in Figure 1. This work quantified the stability of the movement of the particles by changing the phases of the transducers in $1 / 16^{\text {th }}$ wavelength $(8.5 \mathrm{~mm}$ or $13.7 \mathrm{~mm}$ for $40 \mathrm{kHz}$ and $25 \mathrm{kHz}$ respectively) steps, causing the particles to accelerate until they were ejected from the levitation system. The smaller $0.6 \mathrm{~mm}$ particles were confined for accelerations of up to $60 \mathrm{~ms}^{-2}$ corresponding to approximately $500 \mu \mathrm{N}$ of force, whereas the $2 \mathrm{~mm}$ polystyrene particles maintained entrapment up to $30 \mathrm{~ms}^{-2}$ corresponding to approximately $27 \mathrm{mN}$ (both calculated based on $\mathrm{F}=\mathrm{ma}$ ).

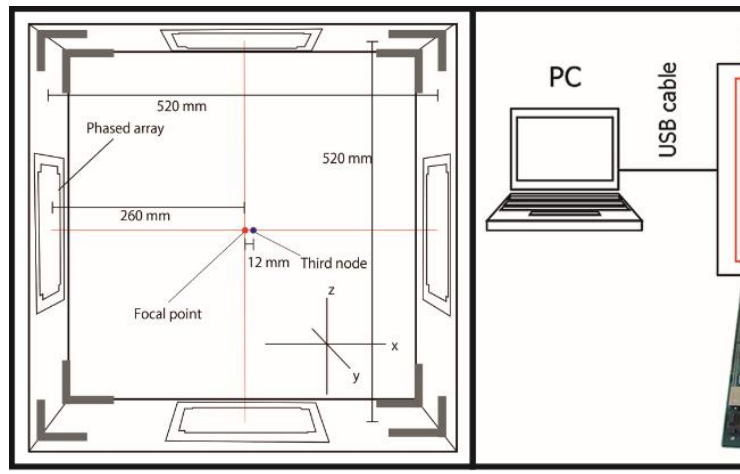

a)

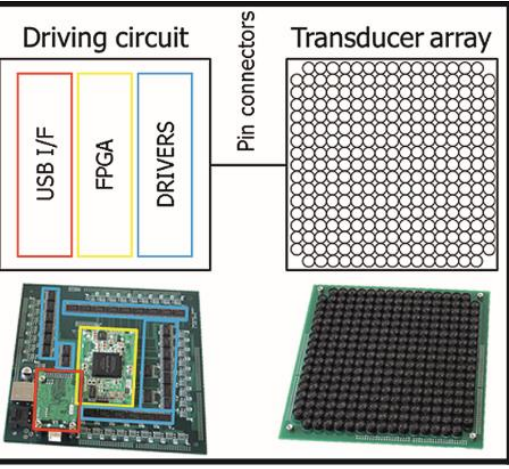

b)

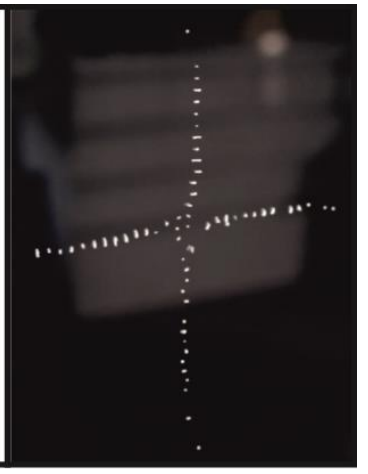

c)

Figure 1. Use of four arrays configured for three-dimensional control of multiple $2 \mathrm{~mm}$ polystyrene spheres. a) shows relative placement of arrays. b) shows the schematic of drive and control electronics. A demonstration of suspension of numerous polystyrene beads using the trapping system is shown in c). Subfigures reproduced from Ochiai $Y$, Hoshi T, Rekimoto J. Three-dimensional mid-air acoustic manipulation by ultrasonic phased arrays. PloS one. 2014 May 21;9(5):e97590. This is an open-access article distributed under the terms of the Creative Commons Attribution License, which permits unrestricted use, distribution, and reproduction in any medium, provided the original author and source are credited.

The concept of an ultrasonic phased array was further developed by Marzo et al. [33] to show that acoustic levitation can be employed to translate, rotate and manipulate particles using a single-sided emitter array. They also introduce a 'holographic acoustic elements framework' that permits the modelling and rapid generation of different traps however their work was still light particle rather than liquid drop based.

Developing this approach, Marzo et al. showed a wide range of different array structures capable of producing what they termed an acoustic tractor beam in their 2017 article Realization of compact tractor beams using acoustic delay-lines [34]. These so-called tractor beams were shown to be capable of holding millimetre-sized polymer particles and even fruit-flies. Figure 2 shows the different methods used by Marzo et al. for generating differing phases from each transducer to produce a focal point for the acoustic field including a physical curved array, flat array with electrically differing phase and flat array with variable tube length in addition to the practical realisation of these methods. In this work it was found that the curved array system was most efficient as the maximum intensity of the transducers was directed toward the target area with minimal losses. 


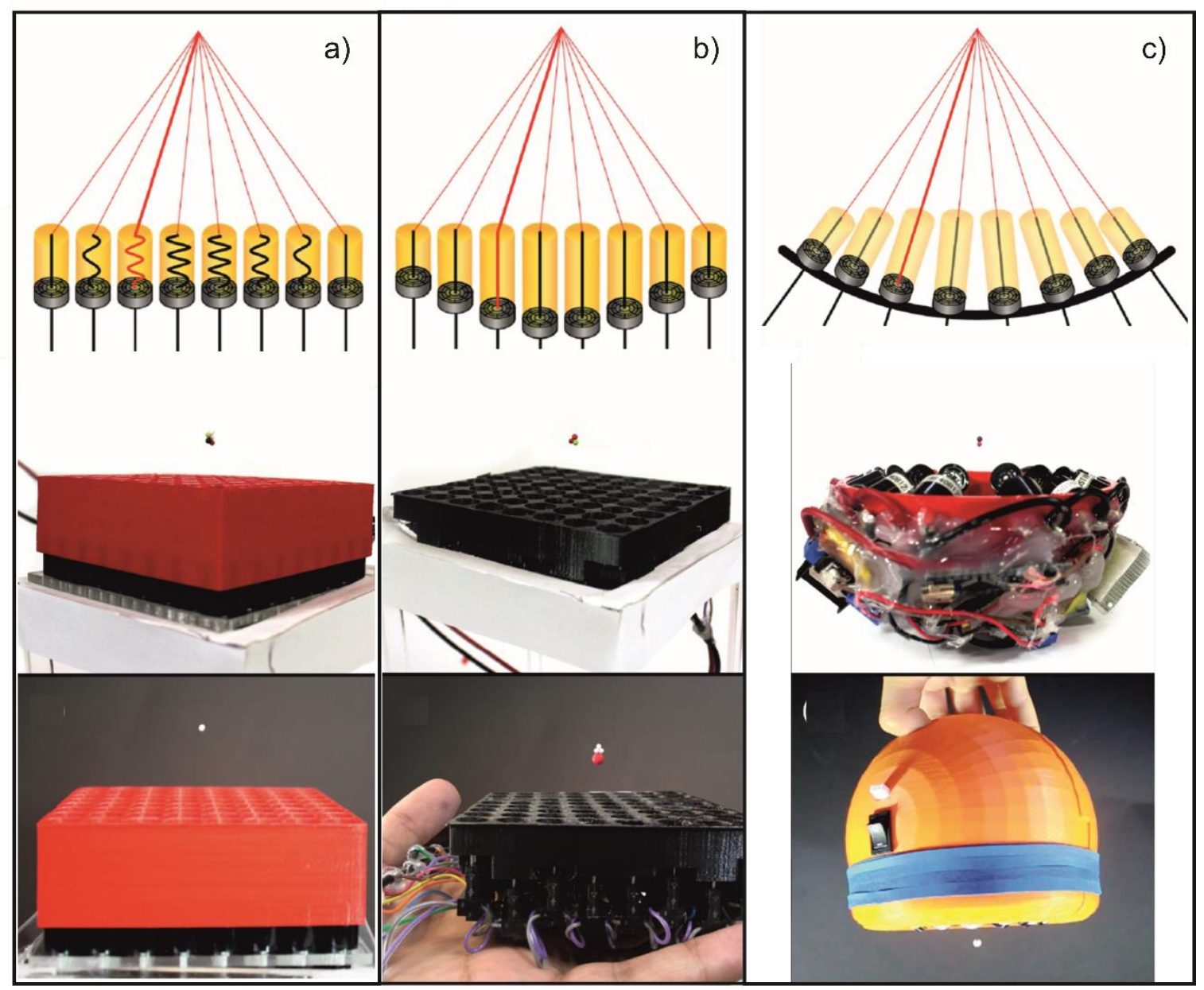

Figure 2 Realization of various compact single sided acoustic levitation devices utilising delay lines. Each panel shows a schematic of the approach at the top and two photographs of its use below. Subfigures (a) have coiled paths to provide phase differences between transducers, (b) are straight tubes of varying length to provide phase differences, whilst (c) is the sculpted surface device where focus is achieved with transducers of similar phases. Subfigures reproduced and rearranged from Marzo A, Ghobrial A, Cox L, Caleap M, Croxford A, Drinkwater BW. Realization of compact tractor beams using acoustic delay-lines. Applied Physics Letters 2017 Jan 2;110(1):014102 with the permission of AIP Publishing.

Simulation work in A method for simultaneous creation of an acoustic trap and a quiet zone [35] showcases a novel method of trap generation in order to create 2 or more simultaneous acoustic traps which levitate light spherical particles. This work builds upon that of Marzo et al. [33], simulating a $16 \times 16$ transducer array which operates at $40 \mathrm{khz}$. These simulations form an acoustic trap at position $r_{1}$ and a "quiet zone" at position $r_{2}$, in which the pressure is comparatively much lower. An acoustic trap at $r_{2}$ and a quiet zone at $r_{1}$ is then superposed which forms 2 acoustic traps with similar strengths and pressure gradients to suspend light particles.

Marzo et al. also developed virtual vortex trapping methods in Acoustic Virtual Vortices with Tuneable Orbital Angular Momentum for Trapping of Mie Particles [36] to explore the effects of orbital angular momentum on the stability of light polystyrene particles within an 
acoustic levitator which suspends particles using a vortex trapping motion. It was also found that particles larger than the wavelength of the incident sound were able to be suspended by switching the driver phases, to make the array emit two different pressure fields. The largest particle which was suspended with reasonable stability was a $16 \mathrm{~mm}$ expanded polystyrene ball, which had a diameter 1.88 times the wavelength of sound, which in this case is $8.6 \mathrm{~mm}$.

Trajectory control of suspended particles is explored in Trajectory Optimization of levitated particles in Mid-air ultrasonic standing wave levitators [37]. In this work an acoustic levitator consisting of 2 opposing planar arrays with 30 transducers on either side, operating at $40 \mathrm{khz}$, was used. Each of the transducers were powered independently and driven with a square wave which had a phase resolution of $\phi=2 \pi / 128$, allowing the focal point to be moved as discussed previously. This entire setup was housed within a chamber upon a passive vibration isolation table which limited external air currents and vibrations respectively. An expanded polystyrene particle was tracked through a circular pathway within the $x-z$ plane, by changing the phases of the transducers to move the focal points. It was however found that the positions in which the particles reached equilibrium were not those that were desired. Corrections to this pathway were applied by comparing the equilibrium position to the target, and it was found that these corrected pathways were the desired shape assuming that the velocity of the particle was less than $1 \mathrm{~cm} / \mathrm{s}$.

Further work by Marzo et al. [38] has explored the capabilities of holographic acoustic tweezers to dynamically manipulate multiple particles simultaneously in mid-air. This is achieved using an algorithm that enables the control of the emitted field from the ultrasonic phased arrays. The two opposing planar arrays consisted of $256,1 \mathrm{~cm}$ diameter transducers on each side, operating at $40 \mathrm{kHz}$. These arrays were separated by $23 \mathrm{~cm}$. The algorithm is used to generate focal points at the position of the particles before controlling the transducer phases to move the foci. The minimum distance between adjacent particles was $1.3 \mathrm{~cm}$, as closer traps merged and inhibited independent control of the 25 total traps. Twin traps were generated in order to control the orientation of asymmetric particles. These were however found to be insufficient to suspend the particles, thus rapid switching between twin traps and focal points was used to orientate and suspend particles.

\section{The liquid levitation era begins}

A major turning point in the application of acoustic levitation was the publication TinyLev: $A$ multi-emitter single-axis acoustic levitator [39] building on the work in [34] but including two curved arrays facing each other. This heralded a new era of acoustic levitation allowing low cost levitation of liquid samples. Whilst still employing the low-cost transducer array, one significant feature of this work was to use low cost Arduino microcontrollers, making it possible for anyone capable of using a soldering iron to produce a viable acoustic levitation system. The details of the design and software were made available in the form of an Instructable [40] bringing levitation to the masses. Fig 3 shows the TinyLev system and examples of levitated objects including liquid drops. This system was able to levitate objects of much higher density than expanded polystyrene balls, including pieces of ceramic, sugar and sapphire spheres. 


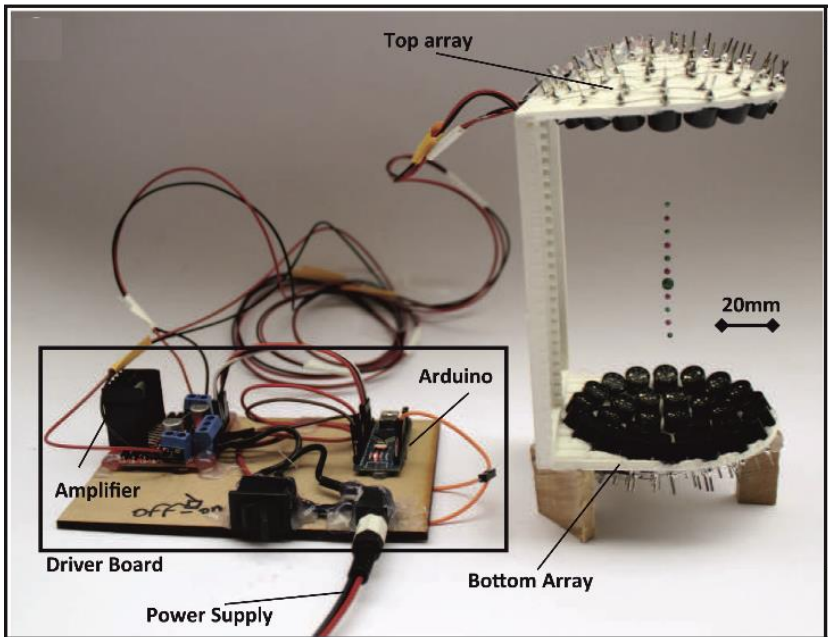

a)

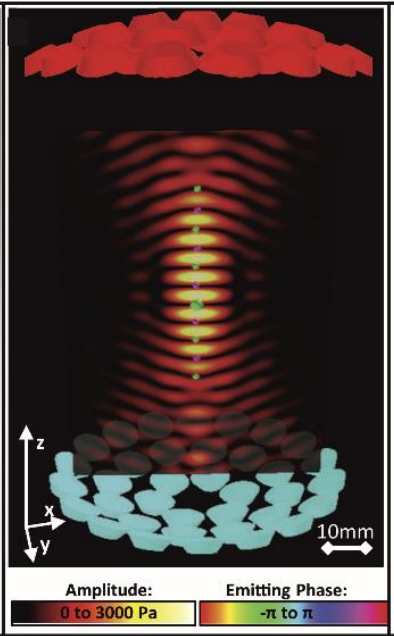

b)

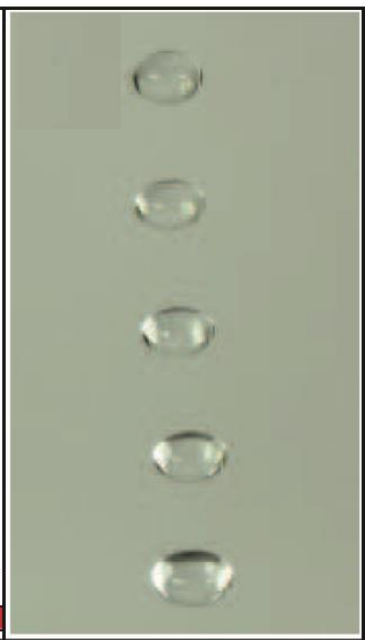

c)

Figure 3. The TinyLev acoustic levitation system, consisting of the driver board and 72 transducers fixed to a $3 D$ printed twin domed structure as shown in a). The simulated acoustic field of such a system is shown in b). Droplets of water suspended by the TinyLev system are shown in c). Note their oblate morphology owing to greater vertical trapping forces than those experienced horizontally. Subfigures reproduced from Marzo A, Barnes A, Drinkwater BW. TinyLev: A multi-emitter single-axis acoustic levitator. Review of Scientific Instruments. 2017 Aug 10;88(8):085105 with the permission of AIP Publishing.

\section{In Ultraino: An Open Phased-Array System for Narrowband Airborne Ultrasound}

Transmission [41], Marzo et al. present a package called Ultraino which they describe as a modular, inexpensive, and open platform that provides hardware, software, and example applications specifically aimed at controlling the transmission of narrowband airborne ultrasound. The aim of this was not only to provide a fixed design example but to allow users to define their own problem and using the supplied modelling software to predict the most appropriate array configuration. Well-defined hardware building blocks can then be used to allow the configuration to be implemented. The realisation that low-cost transducers could be driven by an amplified logic signal with variable phase and duty cycle has transformed the feasibility of phased arrays and in Fig 4 we show examples taken from the Ultraino publication [41]. It should be noted that, even for Ultraino, liquid levitation data was only presented for a two-sided standing wave system similar to the TinyLev and not for any of the single sided configurations which lack the confinement needed for high density samples. One well known phenomenon in single axis acoustic levitation is that the samples are prone to spinning. In many cases this is not significant but where samples are nonspherical, such as insects, or where liquid crystal structure is to be determined this is an important factor which must be considered. 


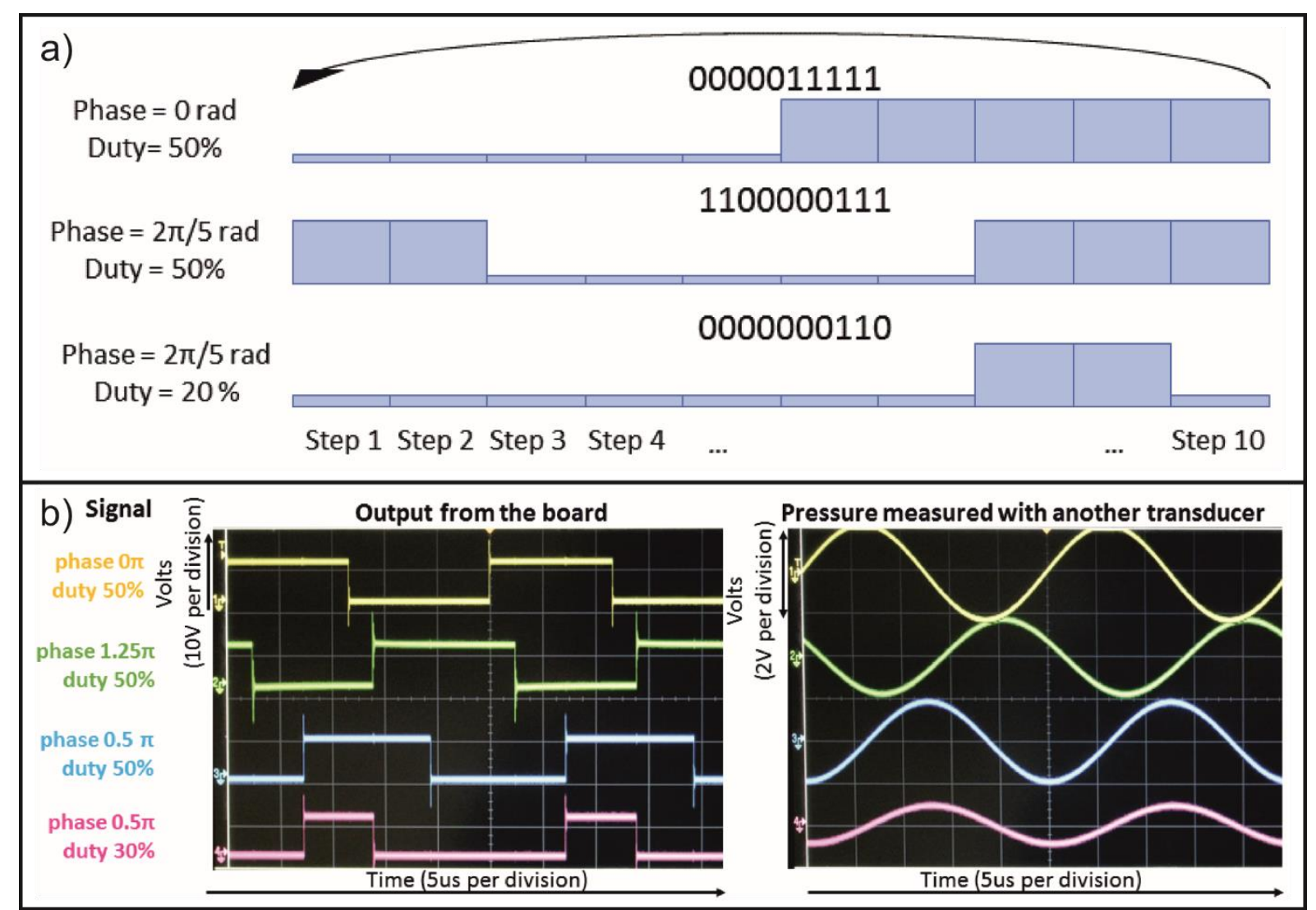

Figure 4. Signals of the Ultraino system. Phases and duty cycles are controlled by a logic signal which is divided into 10 steps as can be seen in a). These signals were recorded from the output of the driver board as can be seen in the left of $b$ ) whilst the responses measured from another transducer which is used as an ultrasonic microphone are shown in the right of b). Subfigures reproduced from Marzo A, Corkett T, Drinkwater BW. Ultraino: An open phased-array system for narrowband airborne ultrasound transmission. IEEE transactions on ultrasonics, ferroelectrics, and frequency control. 2017 Nov 2;65(1):102-11 under Creative Commons Attribution 3.0 License. For more information, see http://creativecommons.org/licenses/by/3.0/

In Acoustic Lock: Position and orientation trapping of non-spherical sub-wavelength particles in mid-air using a single-axis acoustic levitator [42] a variation on the single axis levitator system was reported that saw each transducer 'bowl' divided into two symmetric halves with an invertible phase to facilitate the emission of both vertical standing waves and twintraps, where the confining force is also applied laterally. It was shown that the system could stop the rotation in a supplementary video showing the effect on solid objects and by way of example insects. There is no data presented within the manuscript for liquid levitation although it has been shown in the supplementary material of this work. This system provides the ability to trade off the lateral stability for the levitation of denser materials. Thus, lower density samples, such as insects, may be held in a more stable position than samples such as acrylic or wooden cuboids. 


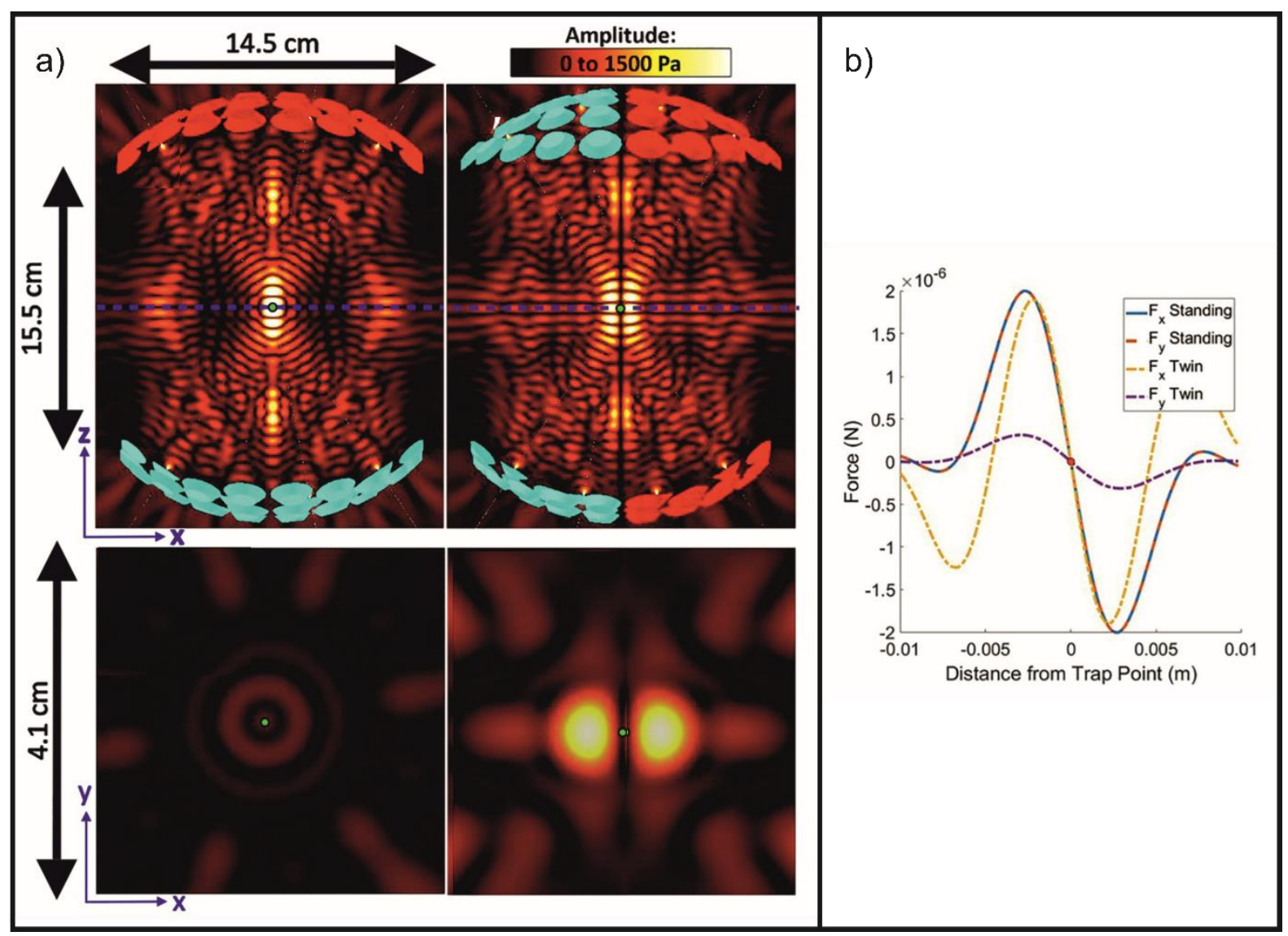

Figure 5. The acoustic field of the so-called acoustic lock system in its multiplexed states is shown in a) where the left images are the standing wave field and the right images show the twin trap field from the side and above in a plan which transects the central trap. The blue lines indicate the planes of the lower images. The force in the vertical and horizontal directions are shown in $b$ for both of the multiplexed states. The central trap is represented as a red dot. Subplots are reproduced from Cox L, Croxford A, Drinkwater BW, Marzo A. Acoustic lock: Position and orientation trapping of non-spherical sub-wavelength particles in mid-air using a single-axis acoustic levitator. Applied Physics Letters. 2018 Jul 30;113(5):054101 with the permission of AIP Publishing.

\section{From basic hardware to liquid drop applications}

In 'Contactless Fluid Manipulation in Air: Droplet Coalescence and Active Mixing by Acoustic Levitation' [43], a rectangular ultrasonic phased array was combined with a reflector surface to demonstrate contactless coalescence and mixing techniques for droplets in air. The array was designed to have two focal points, generated by switching at $500 \mathrm{~Hz}$ between the two (since all transducers are used to form the two traps). The distance between the two focal points could then be reduced to produce a single large standing wave resulting in coalescence of the droplets within a single trap. Figure 6a shows the estimation of the acoustic potentials, the resulting potentials at the pressure nodes and images of two water drops being brought together and coalescing. 

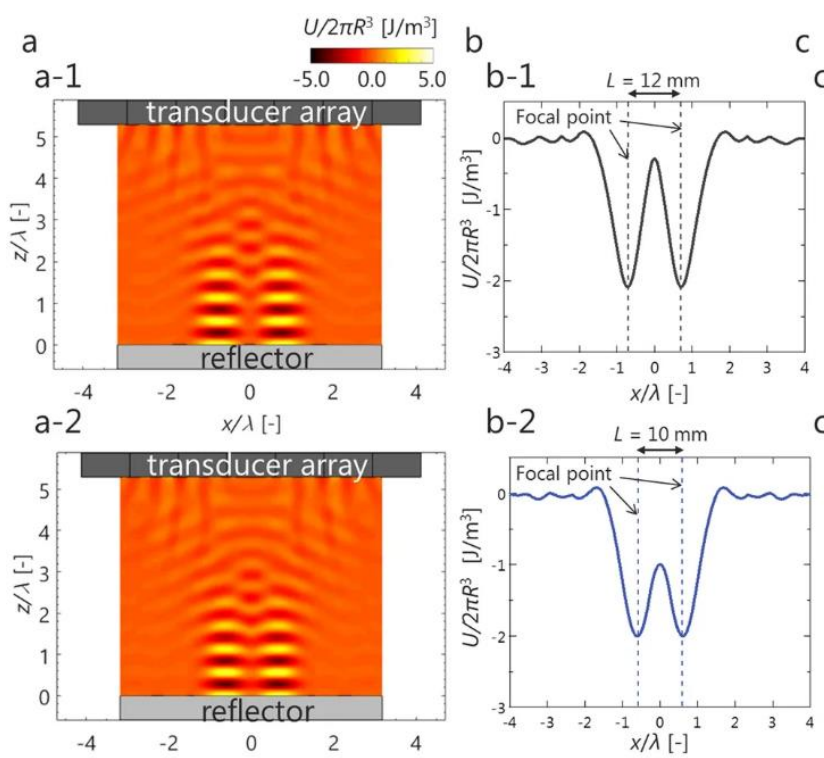

c-1

d
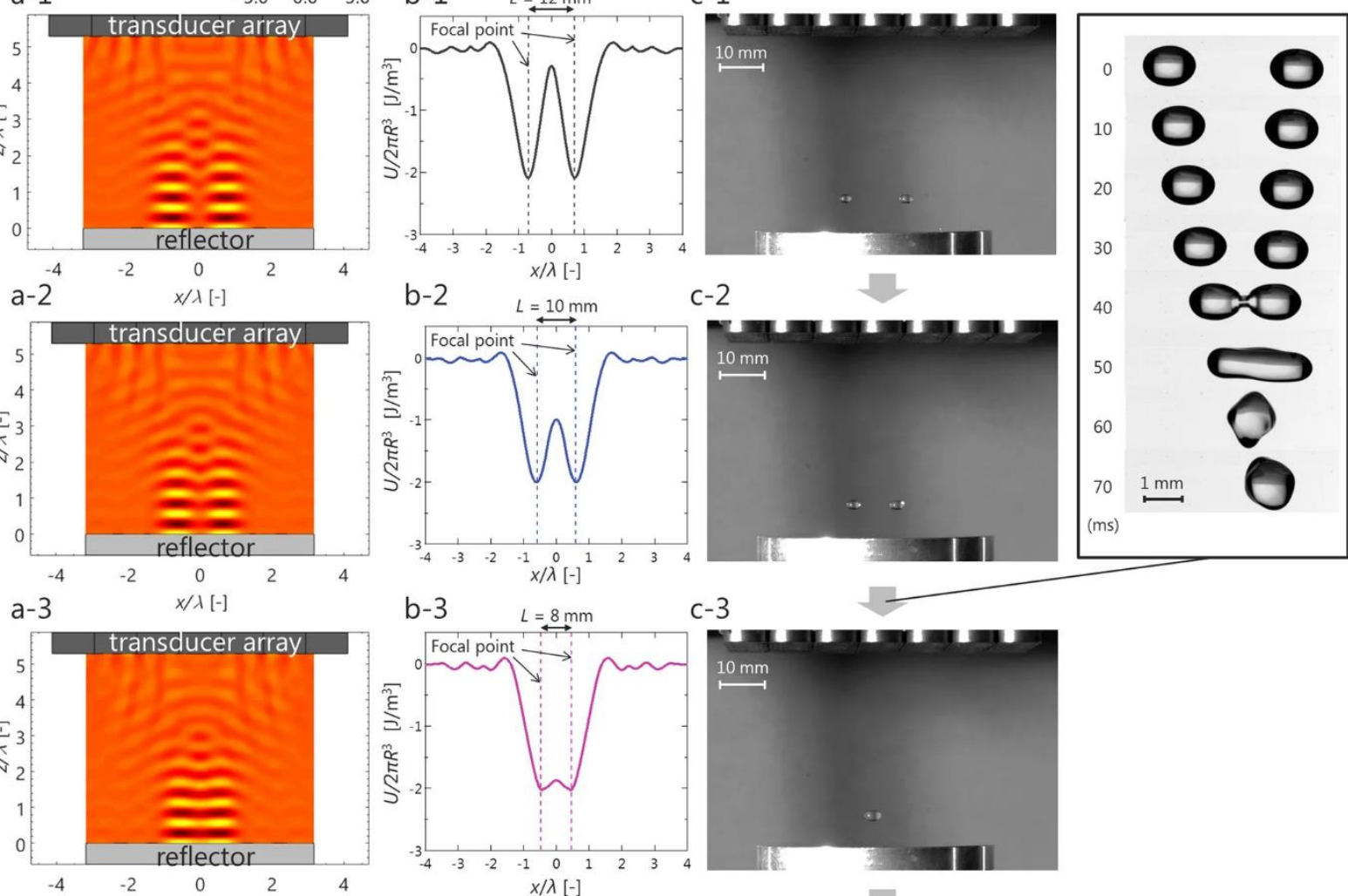

c-3
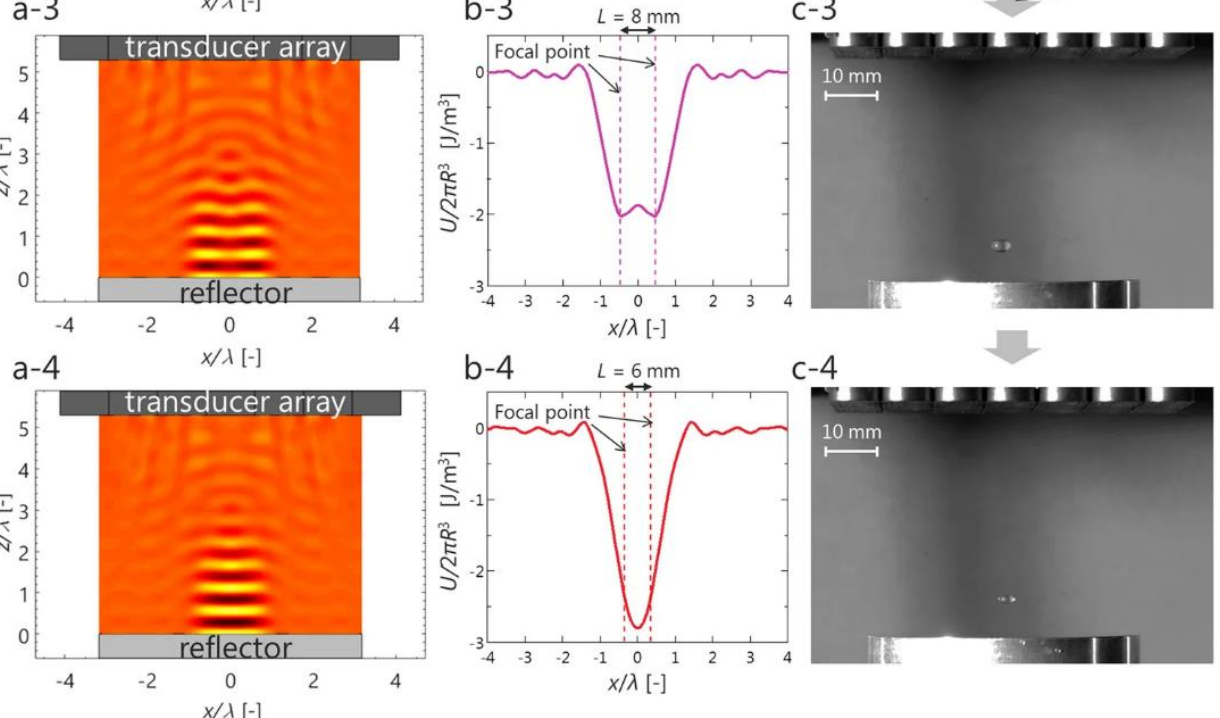

Figure 6. Non-contact translation and coalescence is achieved using time variable acoustic fields. The acoustic potential of the transducer array and reflector combination can be seen in (a), whilst (b) shows the acoustic potential as a function of $x$. Photographs of the droplets within the focal points above the reflector can be seen in (c). A time series of the coalescence is shown in (d). The two focal points are moved toward the central position with each iteration. Reproduced from Watanabe A, Hasegawa K, Abe Y. Contactless fluid manipulation in air: Droplet coalescence and active mixing by acoustic levitation. Scientific reports. 2018 Jul 5;8(1):10221 [43] under a Creative Commons Attribution 4.0 License.

Shen et al. [44] had previously demonstrated oscillation modes in a 'conventional' singleaxis acoustic levitator forming a standing wave between the emitter and the curved reflector by modulating the amplitude by up to $10 \%$. They swept the modulation frequency upward with increments of $0.5 \mathrm{~Hz}$ and observed different oscillation modes being excited. Watanabe [43] implemented a similar scheme in the phased array and compared mixing performance between cases with and without mode oscillation and showed that the flow induced by mode oscillation promotes droplet mixing (an example of which is shown in Figure 6) which effectively brings this technique into a useful tool for containerless chemistry. 
In Automatic contactless injection, transportation, merging, and ejection of droplets with a multifocal point acoustic levitator [45] Andrade et al. used a 16x16 array of $40 \mathrm{kHz}$ ultrasonic transducers, a distance of $110 \mathrm{~mm}$ away from a plane reflector, which along with the superposition of the incident and reflected waves formed a standing wave with a series of pressure nodes where liquid droplets could be trapped and moved in two dimensions above the surface. The reflectors position was chosen by focusing the transducers to different positions and empirically determining which distance provided the largest pressure amplitude. Their system had an integrated droplet injector inserted in the reflector including a piezoelectric buzzer and a $1 \mathrm{~mm}$ diameter nozzle. When a voltage pulse was applied a droplet was injected and trapped at the bottom pressure node of the standing wave. The droplet outlet was a simple hole in the reflector and switching off the acoustic field allowed the drop to pass through under gravity. Figure 7 shows the injection of two droplets followed by the merging and subsequent ejection.

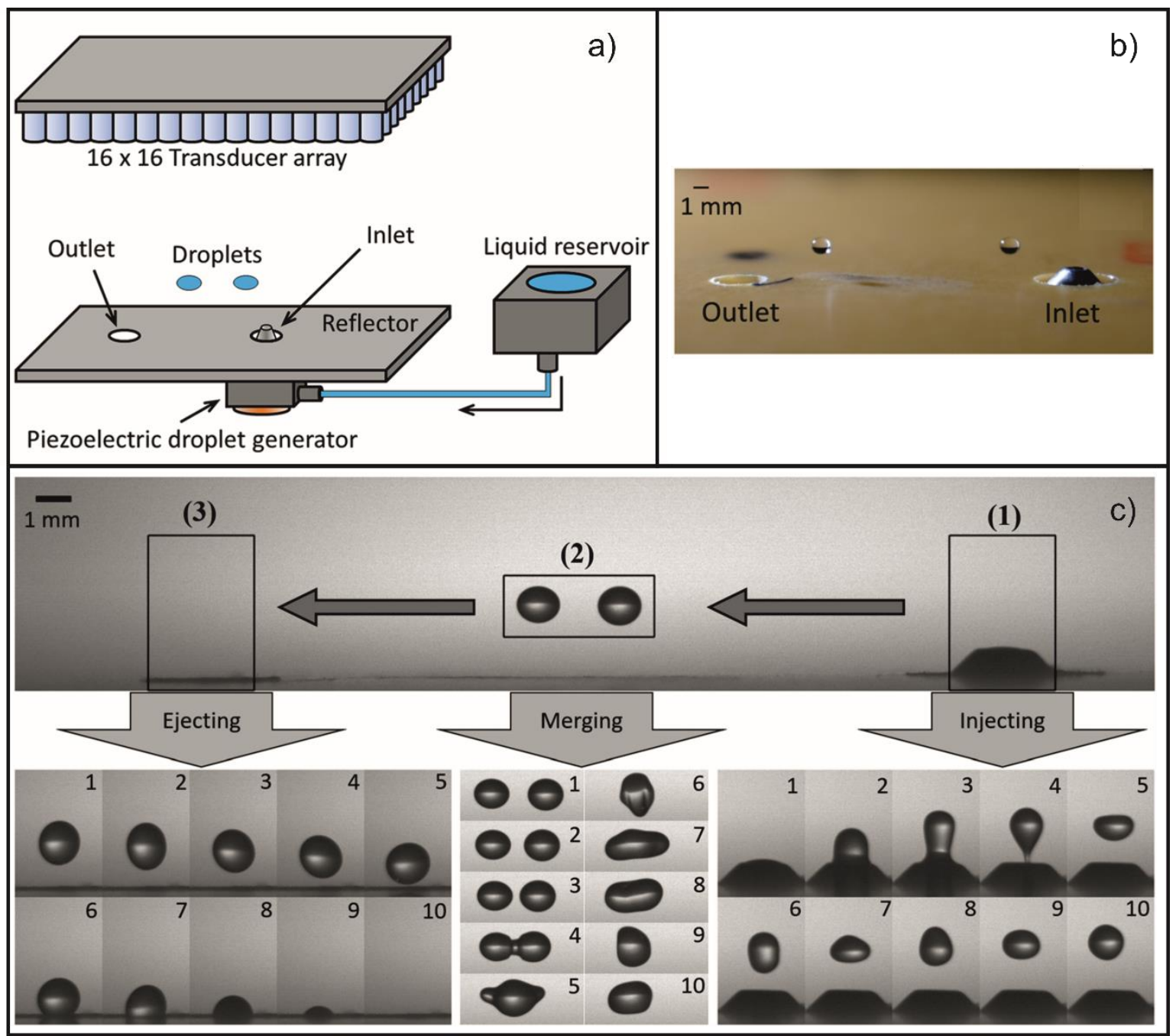

Figure 7. a) Shows a diagram of the acoustic levitation system consisting of $16 \times 16$ transducer array and a planar reflector. Liquid is drawn from the reservoir and into the system via a piezoelectric droplet generator. The droplets are ejected from the system by moving them to above the outlet and switching off the acoustic field. b) shows a colour photograph of the inlet and outlet with two droplets being transported between. c) Shows a series of images of the droplets being injected (1), merged (2) and ejected (3). Subfigures reproduced from Andrade MA, Camargo TS, Marzo A. Automatic contactless injection, transportation, merging, and ejection of droplets with a multifocal point acoustic levitator. Review of Scientific Instruments. 2018 Dec 10;89(12):125105 with the permission of AIP Publishing. 


\section{New Perspectives - The future of acoustic levitation of liquids}

In Acoustic Lock: Position and orientation trapping of non-spherical sub-wavelength particles in mid-air using a single-axis acoustic levitator [42] there was mention in the supplementary material of the levitation of liquids. This made important reference to the shape of the resulting confined droplet: it was found that the droplet formed an approximate ovoid and the boundary of the liquid appeared less smooth. In the move from levitation of solids such as polystyrene balls which have relatively fixed morphology, to fluids which conform to their confinement the shape of the produced acoustic fields has become increasingly important. For many experimental systems, this is simply a feature of the acoustic field which limits the size of droplet that can be confined. In sample presentation scenarios however, the shape of the droplet can be an important factor which directly impacts the results. Consequently, there is an increasing shift from awareness towards control of droplet shape by balancing the confinement potential of the acoustic field against the forces applied to the droplets to maximise sphericity, for example in Non-Contact Universal Sample Presentation for Room Temperature Macromolecular Crystallography Using Acoustic Levitation [50] the droplet sphericity as a function of voltage applied to the TinyLev system was determined and optimised to balance these key parameters.

In recent publications such as [50] this new era of low-cost phased array ultrasonic levitation devices are beginning to find use in sample presentation to non-contact measurement techniques in applications previously making use of Langevin horns [51]. This facilitates containerless, background free spectroscopy which ushers in a new wave of experimental techniques and brings with it significant advantages in terms of measurement resolution without imparting significant energy into the sample. Although further developments are required for these techniques to become universally applied, it is clear that sample presentation systems based on acoustic levitation are likely to become as ubiquitous as pipettes are now in fluidic analysis over the coming decade.

To our knowledge, there are currently no truly single sided systems which can reliably confine non-rigid samples such as fluid droplets, owing to the limited transverse acoustic forces. This represents one of the clear directions for future developments to produce complex acoustic fields which have improved transverse fields for fluid entrapment. Advances in microcontroller systems, capable of smaller wavelength fractions will be a key enabler of such developments, allowing for realistic implementation of arbitrary acoustic fields. The final element which will provide scope for further improvement is the availability of acoustic transducers specifically engineered for such purposes. The limitations in absolute output power, frequency and physical size are largely governed by mass production for other applications such as range finding. Recent developments towards customised transducers such as Measurement and Simulation of an Open-Type Flexural Ultrasonic Transducer [52] will allow for better control of these parameters yielding better control of wavelength and permitting systems to be tailored to specific sample sizes of interest. In combination with sample manipulation processes such as those presented in contactless fluid manipulation in air: Droplet coalescence and active mixing by acoustic levitation such developments will allow for sample preparation and presentation almost all spectroscopic measurement techniques. 


\section{Summary}

In this review article we have summarised the current state of the art of acoustic levitation of liquids using low cost transducer arrays. In this section we briefly summarise the current state of the art for each of the key parameters which dictate the levitation performance. These are then compared to a traditional Langevin system by way of comparison.

\begin{tabular}{|l|l|l|l|l|}
\hline Parameter & Langevin & Ref & Transducer Array Best & Ref \\
\hline Power & $130 \mathrm{~W}$ & {$[53]$} & $5 \mathrm{~W}$ & {$[39]$} \\
\hline Frequency/Wavelength & $25 \mathrm{kHz}$ & {$[54]$} & $28 \mathrm{kHz}, 40 \mathrm{kHz}$ & various \\
\hline Wavelength Step & $\mathrm{n} / \mathrm{a}$ & $\mathrm{n} / \mathrm{a}$ & $2 \pi / 128$ & {$[37]$} \\
\hline Maximum Sample Size & $50 \mathrm{~mm}$ & {$[54]$} & $16 \mathrm{~mm}$ & {$[36]$} \\
\hline Acoustic Force & $12 \mathrm{mN}$ & {$[54]$} & $27 \mathrm{mN}$ & {$[32]$} \\
\hline
\end{tabular}

\section{Conclusion}

The realisation that low-cost transducers could be driven by an amplified logic signal has transformed the feasibility of phased arrays. Through field programmable gate arrays (FPGA) or simple microcontrollers, large numbers of transducers can have their signals tailored in a cost-effective way to produce a given pressure field profile. Commercial companies such as Pixie Dust Technologies [46] and Ultrahaptics [47] offer customers bespoke phased array packages, primarily for mid-air tactile transducers. This review has focused on the development of ultrasonic phased arrays although alongside there have been developments in single transducer levitation through the use of acoustic hologram reflectors [48] and transmission 'metamaterial bricks' [49]. We have presented an expected future direction of the technology based on current direction, but it is clear that we are witnessing the start of a new era of containerless sample preparation and presentation. 


\section{References}

1. Simon MD, Geim AK. Diamagnetic levitation: flying frogs and floating magnets. Journal of applied physics. 2000 May 1;87(9):6200-4.

2. Rhim WK, Chung SK. Containerless protein crystal growth method. Journal of crystal growth. 1991 Mar 1;110(1-2):293-301

3. Ashkin A, Dziedzic JM. Optical levitation by radiation pressure. Applied Physics Letters. 1971 Oct 15;19(8):283-5

4. Winborne DA, Nordine PC, Rosner DE, Marley NF. Aerodynamic levitation technique for containerless high temperature studies on liquid and solid samples. Metallurgical and Materials Transactions B. 1976 Dec 1;7(4):711-3

5. Barmatz M, Collas P. Acoustic radiation potential on a sphere in plane, cylindrical, and spherical standing wave fields. The Journal of the Acoustical Society of America. 1985 Mar;77(3):928-45

6. Brandt EH. Levitation in physics. Science. 1989 Jan 20;243(4889):349-55.

7. Andrade MA, Pérez N, Adamowski JC. Review of Progress in Acoustic Levitation. Brazilian Journal of Physics. 2018 Apr 1;48(2):190-213

8. Santesson S, Nilsson S. Airborne chemistry: acoustic levitation in chemical analysis. Analytical and bioanalytical chemistry. 2004 Apr 1;378(7):1704-9

9. Weber JK, Rey CA, Neuefeind J, Benmore CJ. Acoustic levitator for structure measurements on low temperature liquid droplets. Review of scientific instruments. 2009 Aug 13;80(8):083904

10. Cerenius Y, Oskarsson Å, Santesson S, Nilsson S, Kloo L. Preliminary tests on the use of an acoustic levitator for liquid $\mathrm{X}$-ray diffraction experiments. Journal of applied crystallography. 2003 Feb;36(1):163-4

11. Leiterer J, Delissen F, Emmerling F, Thünemann AF, Panne U. Structure analysis using acoustically levitated droplets. Analytical and bioanalytical chemistry. 2008 Jun 1;391(4):1221-8

12. Leiterer J, Emmerling F, Panne U, Christen W, Rademann K. Tracing coffee tabletop traces. Langmuir. 2008 Jun 26;24(15):7970-8

13. Tsujino $\mathrm{S}$, Tomizaki T. Ultrasonic acoustic levitation for fast frame rate $\mathrm{X}$-ray protein crystallography at room temperature. Scientific reports. 2016 May 6;6:25558

14. Leiterer J, Leitenberger W, Emmerling F, Thünemann AF, Panne U. The use of an acoustic levitator to follow crystallization in small droplets by energy-dispersive X-ray diffraction. Journal of applied crystallography. 2006 Oct 1;39(5):771-3

15. Delißen F, Leiterer J, Bienert R, Emmerling F, Thünemann AF. Agglomeration of proteins in acoustically levitated droplets. Analytical and bioanalytical chemistry. 2008 Sep 1;392(12):161-5

16. Radnik J, Bentrup U, Leiterer J, Brückner A, Emmerling F. Levitated droplets as model system for spray drying of complex oxides: A simultaneous in situ X-ray diffraction/Raman study. Chemistry of Materials. 2011 Dec 6;23(24):5425-31

17. Leopold N, Haberkorn M, Laurell T, Nilsson J, Baena JR, Frank J, Lendl B. On-Line Monitoring of Airborne Chemistry in Levitated Nanodroplets: In Situ Synthesis and Application of SERSActive Ag- Sols for Trace Analysis by FT-Raman Spectroscopy. Analytical chemistry. 2003 May 1;75(9):2166-71

18. Wood BR, Heraud P, Stojkovic S, Morrison D, Beardall J, McNaughton D. A portable Raman acoustic levitation spectroscopic system for the identification and environmental monitoring of algal cells. Analytical chemistry. 2005 Aug 1;77(15):4955-61

19. Biedasek S, Abboud M, Moritz HU, Stammer A. Online-Analysis on Acoustically Levitated Droplets. InMacromolecular symposia 2007 Dec (Vol. 259, No. 1, pp. 390-396). Weinheim: WILEY-VCH Verlag 
20. Cronin JT, Brill TB. Acoustic levitation as an IR spectroscopy sampling technique. Applied Spectroscopy. 1989 Feb;43(2):253-7

21. Brotton SJ, Kaiser RI. Novel high-temperature and pressure-compatible ultrasonic levitator apparatus coupled to Raman and Fourier transform infrared spectrometers. Review of Scientific Instruments. 2013 May 29;84(5):055114

22. Brotton SJ, Lucas M, Jensen TN, Anderson SL, Kaiser RI. Spectroscopic Study on the Intermediates and Reaction Rates in the Oxidation of Levitated Droplets of Energetic lonic Liquids by Nitrogen Dioxide. The Journal of Physical Chemistry A. 2018 Aug 3;122(37):735177

23. Brotton SJ, Kaiser RI. Spectroscopic Study on the Polymer Condensates Formed via Pyrolysis of Levitated Droplets of Dicyanamide-Containing Ionic Liquids. The Journal of Physical Chemistry A. 2019 Feb 4

24. Brotton SJ, Lucas M, Chambreau SD, Vaghjiani GL, Yu J, Anderson SL, Kaiser RI. Spectroscopic investigation of the primary reaction Intermediates in the oxidation of levitated droplets of energetic ionic liquids. The journal of physical chemistry letters. 2017 Dec 5;8(24):6053-9

25. Benmore CJ, Weber JK, Tailor AN, Cherry BR, Yarger JL, Mou Q, Weber W, Neuefeind J, Byrn SR. Structural characterization and aging of glassy pharmaceuticals made using acoustic levitation. Journal of pharmaceutical sciences. 2013 Apr 1;102(4):1290-300

26. Leiterer J, Grabolle M, Rurack K, Resch-Genger U, Ziegler J, Nann T, Panne U. Acoustically levitated droplets. Annals of the New York Academy of Sciences. 2008 May 1;1130(1):78-84

27. Warschat C, Stindt A, Panne U, Riedel J. Mass spectrometry of levitated droplets by thermally unconfined infrared-laser desorption. Analytical chemistry. 2015 Aug 3;87(16):8323-7

28. Westphall MS, Jorabchi K, Smith LM. Mass spectrometry of acoustically levitated droplets. Analytical chemistry. 2008 Jun 27;80(15):5847-53

29. Crawford EA, Esen C, Volmer DA. Real time monitoring of containerless microreactions in acoustically levitated droplets via ambient ionization mass spectrometry. Analytical chemistry. 2016 Aug 16;88(17):8396-403

30. Hoshi T, Takahashi M, Iwamoto T, Shinoda H. Noncontact tactile display based on radiation pressure of airborne ultrasound. IEEE Transactions on Haptics. 2010 Jul;3(3):155-65

31. Hoshi T, Ochiai Y, Rekimoto J. Three-dimensional noncontact manipulation by opposite ultrasonic phased arrays. Japanese Journal of Applied Physics. 2014 Jun 12;53(7S):07KE07

32. Ochiai Y, Hoshi T, Rekimoto J. Three-dimensional mid-air acoustic manipulation by ultrasonic phased arrays. PloS one. 2014 May 21;9(5):e97590

33. Marzo A, Seah SA, Drinkwater BW, Sahoo DR, Long B, Subramanian S. Holographic acoustic elements for manipulation of levitated objects. Nature communications. 2015 Oct 27;6:8661

34. Marzo A, Ghobrial A, Cox L, Caleap M, Croxford A, Drinkwater BW. Realization of compact tractor beams using acoustic delay-lines. Applied Physics Letters. 2017 Jan 2;110(1):014102

35. Andersson C, Ahrens J. A Method for Simultaneous Creation of an Acoustic Trap and a Quiet Zone. In2018 IEEE 10th Sensor Array and Multichannel Signal Processing Workshop (SAM) 2018 Jul 8 (pp. 622-626)

36. Marzo A, Caleap M, Drinkwater BW. Acoustic virtual vortices with tunable orbital angular momentum for trapping of mie particles. Physical review letters. 2018 Jan 22;120(4):044301

37. Fushimi T, Marzo A, Hill TL, Drinkwater BW. Trajectory Optimization of Levitated Particles in Mid-Air Ultrasonic Standing Wave Levitators. In2018 IEEE International Ultrasonics Symposium (IUS) 2018 Oct 22 (pp. 1-9)

38. Marzo A, Drinkwater BW. Holographic acoustic tweezers. Proceedings of the National Academy of Sciences. 2019 Jan 2;116(1):84-9

39. Marzo A, Barnes A, Drinkwater BW. TinyLev: A multi-emitter single-axis acoustic levitator. Review of Scientific Instruments. 2017 Aug 10;88(8):085105

40. UpnaLab (2019). Acoustic Levitator. [online] Instructables.com. Available at: https://www.instructables.com/id/Acoustic-Levitator/ [Accessed 27 Jun. 2019]. 
41. Marzo A, Corkett T, Drinkwater BW. Ultraino: An open phased-array system for narrowband airborne ultrasound transmission. IEEE transactions on ultrasonics, ferroelectrics, and frequency control. 2017 Nov 2;65(1):102-11

42. Cox L, Croxford A, Drinkwater BW, Marzo A. Acoustic lock: Position and orientation trapping of non-spherical sub-wavelength particles in mid-air using a single-axis acoustic levitator. Applied Physics Letters. 2018 Jul 30;113(5):054101

43. Watanabe A, Hasegawa K, Abe Y. Contactless fluid manipulation in air: Droplet coalescence and active mixing by acoustic levitation. Scientific reports. $2018 \mathrm{Jul}$ 5;8(1):10221

44. Shen $\mathrm{CL}$, Xie WJ, Wei B. Parametrically excited sectorial oscillation of liquid drops floating in ultrasound. Physical Review E. 2010 Apr 9;81(4):046305

45. Andrade MA, Camargo TS, Marzo A. Automatic contactless injection, transportation, merging, and ejection of droplets with a multifocal point acoustic levitator. Review of Scientific Instruments. 2018 Dec 10;89(12):125105

46. Pixie Dust Technologies (2019). Project. [online] ピクシーダストテクノロジーズ株式会社 (Pixy Dust Technologies Co., Ltd.). Available at: https://pixiedusttech.com/project/ [Accessed 27 Jun. 2019].

47. Ultrahaptics. (2019). Ultrahaptics - Discover a new type of haptics. [online] Available at: https://www.ultrahaptics.com/products-programs/stratos-explore-development-kit/ [Accessed 27 Jun. 2019].

48. Melde K, Mark AG, Qiu T, Fischer P. Holograms for acoustics. Nature. 2016 Sep;537(7621):518.

49. Memoli G, Caleap M, Asakawa M, Sahoo DR, Drinkwater BW, Subramanian S. Metamaterial bricks and quantization of meta-surfaces. Nature communications. 2017 Feb 27;8:14608.

50. Morris RH, Dye ER, Axford DA, Newton MI, Beale J, Docker P. Non-Contact Universal Sample Presentation for Room Temperature Macromolecular Crystallography Using Acoustic Levitation. Scientific reports. 2019. Under Review

51. Tsujino S, Shinoda A, Tomizaki T. On-demand droplet loading of ultrasonic acoustic levitator and its application for protein crystallography experiments. 2019. Appl. Phys. Lett. 114, 213702

52. Acoustofluidics

53. https://pdfs.semanticscholar.org/94fe/90ef5ed87c202b8a2f99e9e974d70804da05.pdf

54. https://pureapps2.hw.ac.uk/ws/portalfiles/portal/10765644/pdf archiveAPPLABvol 109iss 40441011 am.pdf 